\title{
Etude de l'exposition industrielle à des aérosols d'uranium dans le procédé d'enrichissement par laser. Méthodes et résultats
}

\author{
E. ANSOBORLO*, M. CLARAZ*, M.H. HENGE-NAPOLI*, H. MÉTIVIER*, \\ J. GARDEN**, M.C. CHEYNET**
}

(Manuscrit reçu le 21 janvier 1994,

révisé le 1er août 1994, accepté le 15 décembre 1994)

RÉSUMÉ Le développement en France d'un nouveau procédé d'enrichissement par laser de l'uranium nous a conduits à étudier en situation industrielle les risques radio-

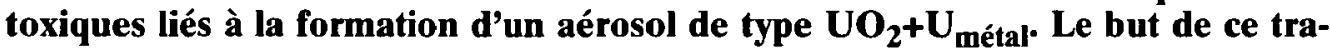
vail est de déterminer pour ce mélange d'oxydes d'uranium les paramètres physico-chimiques et biocinétiques spécifiques à ce composé et nécessaires ultérieurement pour le calcul de dose, ceci en fonction du nouveau modèle pulmonaire décrit par la CIPR 66. Les résultats concernant les mesures de diamètre aérodynamique médian en activité (DAMA) donnent des valeurs comprises entre 5,2 et $10 \mu \mathrm{m}$ avec jusqu'à $20 \%$ de particules inférieures à $1 \mu \mathrm{m}$, tandis que les concentrations au poste de travail varient de 1,8 à $125 \mathrm{~Bq} \mathrm{~m}^{-3}$ et le calcul des périodes biologiques conduit à $48 \mathrm{j}$ pour les tests de dissolution in vitro et à $77 \mathbf{j}$ pour les expériences d'inhalation in vivo, les valeurs de taux de dissolution obtenues permettent d'affirmer que ce composé est de type W suivant la classification de la CIPR 30 et de type M suivant la CIPR 66.

ABSTRACT Comprehensive studies of the radiotoxicological risk at new uranium enrichment processing facilities using laser isotopic separation, were particularly motivated by the generation of a uranium oxide aerosol identified as $\mathrm{UO}_{2}+\mathrm{U}_{\text {metal }}$. Taking the new ICRP 66 recommendations into account, the following study on this uranium oxide mixture, was aimed at determining the physico-chemical and biokinetic specific parameters required in order to calculate the effective dose. The activity median aerodynamic diameters (AMAD) ranged between 5.2 and $10 \mu \mathrm{m}$ with, in some cases, up to $20 \%$ of submicron size particles, while concentration values at the workplace ranged from 1.8 to $125 \mathrm{~Bq} \mathrm{~m}^{-3}$ and biological half-time calculations gave a 48 d period with in vitro dissolution test and a 77 d period with in vivo inhalation experiments. Transfer rates and dissolution rates obtained from both in vitro and in vivo experiments intend to emphasize a class $W$ behaviour in term of ICRP 30 and $M$ in term of ICRP 66.

\footnotetext{
* Institut de protection et de sureté nucléaire, DPHD/SDOS/LEAR, BP 38, 26701 Pierrelatte, France.

** Institut national polytechnique de Grenoble, ENSEEG/CMTC, BP 75, 38402 St Martin d'Hères, France.
} 


\section{Introduction}

Un nouveau procédé d'enrichissement de l'uranium, dont le principe est la séparation isotopique par laser de l'uranium en phase vapeur atomique, ou procédé SILVA, est actuellement développé en France sur les centres d'études nucléaires de Saclay et de Pierrelatte. Lors du nettoyage des structures, après vaporisation dans les enceintes pilotes, les travailleurs vêtus de combinaisons et équipés de protection respiratoire peuvent être exposés, en cas d'incidents, à des aérosols d'oxyde d'uranium.

Le but de cette étude sera donc de caractériser sur le plan physico-chimique et biologique les composés rencontrés et d'évaluer les paramètres expérimentaux nécessaires pour le calcul de dose dans le cadre du nouveau modèle pulmonaire de la CIPR 66 [12], ceci afin d'optimiser la protection des travailleurs. Le risque d'exposition à des actinides nous a conduit à développer, pour certains composés de l'uranium [1-3], une méthodologie d'étude particulièrement guidée par les recommandations de la CIPR 66 [12], et dont les principales étapes sont :

1) la mesure de la concentration en $\mathrm{Bq} \mathrm{m}^{-3}$ et la distribution granulométrique exprimée en $D A M A^{1}$ au niveau du poste de travail ;

2) la caractérisation physico-chimique de ces aérosols ;

3) la détermination in vitro de leur solubilité chimique ou cellulaire ;

4) l'étude de leur biocinétique in vivo après inhalation par des rats.

Les données expérimentales ainsi obtenues vont permettre de déterminer les paramètres suivants, spécifiques du composé industriel manipulé, à savoir : la densité, le ou les $D A M A$ mesurés au poste de travail et les paramètres de dissolution exprimés en période de demi-vie, en pourcentage dissous à $24 \mathrm{~h}$, en fraction rapidement éliminée $f_{\mathrm{r}}$ et en taux de transfert sanguin journalier $s_{\mathrm{r}}$ et $s_{\mathrm{S}}$ tels qu'ils sont définis dans le nouveau modèle pulmonaire.

De nombreuses données bibliographiques concernant les oxydes industriels du type $\mathrm{UO}_{2}$ et $\mathrm{U}_{3} \mathrm{O}_{8}$ existent : ainsi des expérimentations in vivo chez l'homme ou l'animal $[1,14,16,19-20]$ donnent des périodes biologiques entre 120 et 360 j pour $\mathrm{U}_{3} \mathrm{O}_{8}$ et un coefficient de transfert $F_{\mathrm{b}}{ }^{2}$ compris entre 1 et $1,7 \times 10^{-3} \mathrm{j}^{-1}[18,20]$ et des périodes entre 109 et $390 \mathrm{j}$ pour $\mathrm{UO}_{2}$ avec $F_{\mathrm{b}}$ compris entre 2,6 et $6 \times 10^{-4} \mathrm{j}^{-1}$ [19] ; les études in vitro $[7,8,13,21]$ ont montré que les périodes de dissolution étaient de 50 à $500 \mathrm{j}$ pour $\mathrm{U}_{3} \mathrm{O}_{8}$ et 200 à $500 \mathrm{j}$ pour $\mathrm{UO}_{2}$.

1. $D A M A=$ diamètre aérodynamique médian en activité.

2. $F_{\mathrm{b}}=\left(0,693 / T_{1 / 2}\right) \cdot(z / y)$ où $T_{1 / 2}$ est la période biologique, $z$ le pourcentage de dépôt initial transféré dans le sang et $y$ le pourcentage de dépôt initial éliminé du poumon. 
Des recherches préliminaires sur la composition des aérosols émis ont permis d'identifier un mélange de type $\mathrm{UO}_{2}+\mathrm{U}_{\text {métal }}$ avec des traces d' $\mathrm{U}_{3} \mathrm{O}_{8}$. En ce qui concerne les aérosols d'uranium métal ou d'un mélange $\mathrm{UO}_{2}+\mathrm{U}_{\text {métal }}$ rencontrés dans ce procédé, peu d'informations sont disponibles : quelques résultats sur la taille des particules provenant d'opérations métallurgiques sur de l'uranium métal sont données par Hyatt [10] avec des DAMA compris entre 0,4 et $3,9 \mu \mathrm{m}$ et une valeur plus faible de $0,05 \mu \mathrm{m}$ dans le cas de fumées; quelques mesures de charge pulmonaire chez des travailleurs exposés lors d'opérations de perçage ou de frittage de lingots d'uranium [17] avec 3 périodes de 7 j $(10 \%), 70$ j $(53 \%)$ et 390 j (7\%) ; enfin, des données sur le métabolisme de l' $\mathrm{U}_{3} \mathrm{O}_{8}$ après une opération de fonte d'uranium [18] conduisent au calcul des 3 périodes suivantes : 1 j $(25 \%), 4$ j $(25 \%)$ et 210 j $(50 \%)$ et un coefficient de transfert $F_{\mathrm{b}}$ de $10^{-3} \mathrm{j}^{-1}$.

Ce document présente les paramètres expérimentaux obtenus pour le composé $\mathrm{UO}_{2}+\mathrm{U}_{\text {métal }}$.

\section{Matériel et méthodes}

Cette étude a été réalisée sur les installations pilotes du site de Pierrelatte, d'une part dans l'atelier HORUS, lors de l'ouverture de l'évaporateur et de la manipulation de l'enceinte collectrice, d'autre part dans l'atelier ARUS, lors de la récupération de l'uranium par grattage des éléments de l'enceinte et lors du nettoyage des structures. La caractérisation physico-chimique et la biocinétique de ce composé ont été étudiées selon la méthodologie suivante :

\subsection{Prélèvements atmosphériques et granulométrie}

Les prélèvements individuels sont effectués à l'aide de pompes GILAIR suivant la norme NF X 43-271, sur membrane filtrante millipore $0,8 \mu \mathrm{m}$ à un débit de $11 \mathrm{~min}^{-1}$. Les prélèvements en ambiance ou près de la source d'émission sont faits sur filtres identiques mais avec un débit de $301 \mathrm{~min}^{-1}$. Les analyses de filtres sont réalisées par comptage $\alpha$ et exprimées en $\mathrm{Bq}$ et les résultats de concentration ainsi obtenus sont calculés en $\mathrm{Bq} \mathrm{m}^{-3}$.

La distribution granulométrique est réalisée à l'aide soit d'un impacteur en cascade Andersen à 8 étages $(0,4$ à $9 \mu \mathrm{m})$ avec un débit de $281 \mathrm{~min}^{-1}$, soit d'un impacteur Andersen basse pression (114 mm Hg) à 13 étages $(0,08$ à $35 \mu \mathrm{m})$ avec un débit de $2,51 \mathrm{~min}^{-1}$. Les résultats sont exprimés sous forme de distribution en activité avec détermination du $D A M A$ exprimé en $\mu \mathrm{m}$ ainsi que l'écart-type géométrique $\sigma g$. Des photos ont été prises par microscopie électronique à balayage (MEB) (JEOL 6400) afin de visualiser les particules déposées sur les filtres. 


\subsection{Caractéristiques physico-chimiques}

Les mesures de densité sont effectuées par picnométrie et la surface spécifique est déterminée par la méthode du BET. Le composé manipulé sur HORUS et ARUS a été caractérisé à l'aide de diverses techniques telles que la diffraction des rayons X (Philips PW 1730), l'infra-rouge solide (Perkin Elmer 1760 ) et la microscopie électronique à balayage en transmission (MEBT-VG/HB 501) couplée à un spectromètre de perte d'énergie des électrons.

\subsection{Dissolution in vitro}

Les tests de dissolution in vitro chimique et cellulaire retenus pour cette étude, utilisant le milieu de culture cellulaire (199 Sigma) et des macrophages alvéolaires de rats ont été décrits précédemment [4]. L'objectif de ces tests est de calculer les périodes de dissolution du composé $\mathrm{UO}_{2}+\mathrm{U}_{\text {métal }}$, les pourcentages dissous à $24 \mathrm{~h}$ ainsi que les taux de transfert journalier, et d'interpréter l'effet des macrophages dans le phénomène de solubilisation.

\subsection{Etudes in vivo}

Trois séries de campagnes d'inhalation ont été effectuées, chacune sur 14 rats Sprague Dawley (IFFA CREDO, Lyon). La poussière d' $\mathrm{UO}_{2}+\mathrm{U}_{\text {métal }}$ prélevée au poste de travail a été préséparée par une sédimentation de $30 \mathrm{~min}$ dans l'alcool. La génération des aérosols dans la chambre d'inhalation (Ysebaert, France) est faite à partir d'une suspension aqueuse de la poussière dans un nébuliseur. Les animaux sont exposés entre $45 \mathrm{~min}$ et $1 \mathrm{~h}$ et la concentration en uranium dans la chambre est comprise entre 0,35 et $0,45 \mathrm{~Bq} \mathrm{~m}^{-3}$ et le $D A M A$ entre 2,2 et $3,5 \mu \mathrm{m}$ avec $\sigma g>2,5$.

Après chaque inhalation, 2 animaux sont sacrifiés pour mesurer le dépôt initial pulmonaire $(D I P)$; les autres animaux sont mis en cage à métabolisme et sacrifiés dans le temps. Les urines sont recueillies journellement et différents organes, tels que reins, carcasse ou fémur sont analysés. Le but principal est de calculer les périodes de demi-vie biologique et les coefficients de transfert sanguin journalier $F_{\mathrm{b}}$ et de comparer les résultats obtenus in vivo avec ceux de l'expérimentation in vitro.

Une instillation intratrachéale a également été pratiquée sur des rats pour étudier le comportement intracellulaire du mélange $\mathrm{UO}_{2}+\mathrm{U}_{\text {métal }}$. Après sacrifice aux temps $t=1 \mathrm{j}, 4 \mathrm{j}, 9 \mathrm{j}$, et $20 \mathrm{j}$ un lavage bronchoalvéolaire a permis de récupérer les macrophages alvéolaires pour une observation microscopique au MEBT. 


\section{Résultats}

\subsection{Concentrations atmosphériques et granulométrie des aérosols au poste de travail}

Vingt campagnes de prélèvements sur les postes HORUS et ARUS ont révélé des concentrations individuelles comprises entre 0,2 et $18,5 \mathrm{~Bq} \mathrm{~m}^{-3}$ et des résultats d'ambiance près de la source d'émission lors des opérations de grattage entre 1,8 et $125,7 \mathrm{~Bq} \mathrm{~m}^{-3}$ (Fig. 1). Le DAMA de ces aérosols varie entre 5,2 et $10,0 \mu \mathrm{m}$ avec un $\sigma g$ compris entre 1,5 et 3,6. L'uranium métal recouvrant la surface des structures sous forme plus ou moins oxydée est très pyrophorique : lors des manipulations de grattage quelques petits feux sont initiés, conduisant à la formation de fumées semblables à celles observées au niveau des postes de soudure, et pouvant contenir jusqu'à $20 \%$ de particules de taille inférieure à $1 \mu \mathrm{m}$ (Fig. 2).

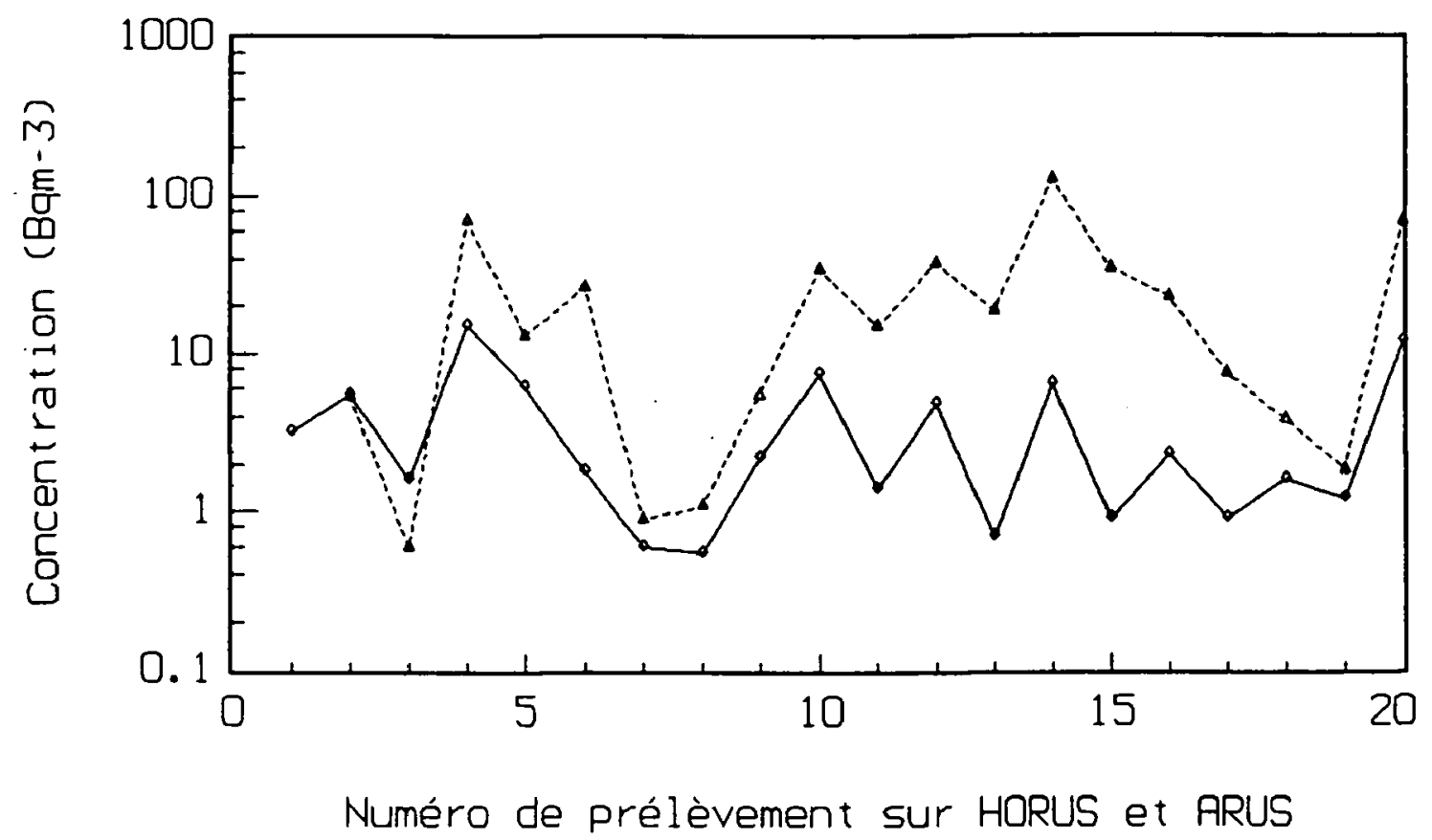

Fig. 1 - Résultats des 20 campagnes de prélèvements sur le composé $\mathrm{UO}_{2}+U_{\text {métal }}$ effectuées sur HORUS et ARUS et exprimées en concentration individuelle ou à la source.

Results of 20 sampling series on $\mathrm{UO}_{2}+U_{\text {metal }}$ carried out on HORUS and ARUS expressed in individual concentrations or at the source.

\subsection{Caractéristiques physico-chimiques}

La densité mesurée est de $7,2 \mathrm{~g} \mathrm{~cm}^{-3}$ et la surface spécifique de $1 \mathrm{~m}^{2} \mathrm{~g}^{-1}$. Les spectres de diffraction des rayons $\mathrm{X}$ ainsi que l'infra-rouge solide ont permis d'identifier l'uranium métal et l'oxyde d'uranium. L'analyse en perte d'énergie sur plusieurs particules a mis en évidence la présence d'uranium métal au cœur des particules avec une oxydation à la surface caractérisée par les raies $\mathrm{O}_{4,5}$ d'énergie de l'uranium à $96 \mathrm{eV}$ et $\mathrm{K}$ de l'oxygène à $531 \mathrm{eV}$. Dans le cas de petits feux d'uranium, des traces d' $\mathrm{U}_{3} \mathrm{O}_{8}$ sont mises en évidence. 


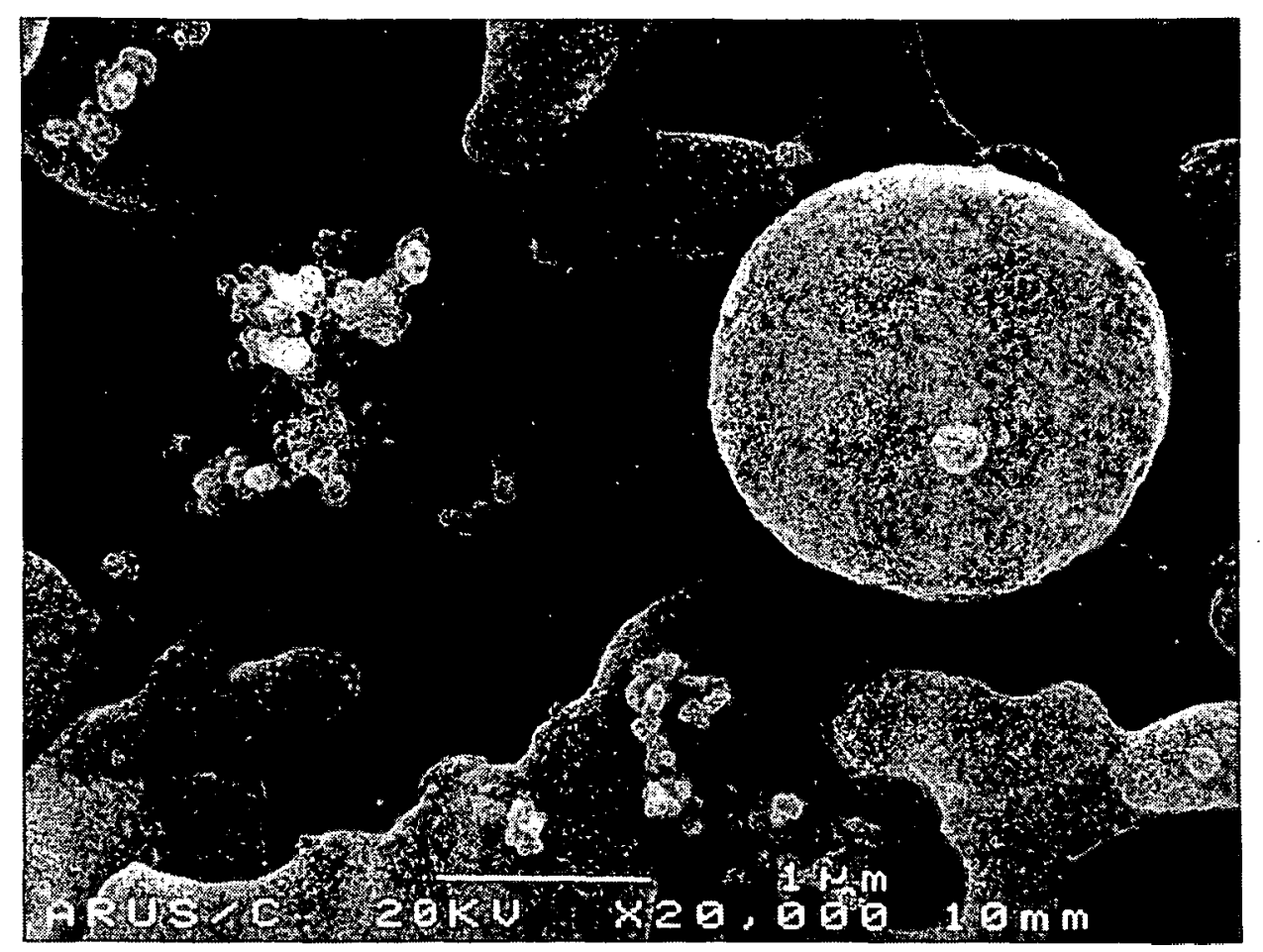

Fig. 2 - Particules d'uranium observées au microscope électronique à balayage $(G=20$ 000) lors de l'opération de grattage sur le poste ARUS.

$U$ particles observed with a scanning electron microscope $(G=20000)$ during scraping at the ARUS station.

\subsection{Dissolution in vitro}

La cinétique de dissolution chimique, réalisée sur 15 jours en milieu de culture cellulaire, est décrite comme la somme de deux exponentielles avec des demi-vies de $1 \mathrm{j}(26 \%)$ et $48 \mathrm{j}$ (74 \%). La comparaison des tests chimique et cellulaire sur un temps court $(3 \mathrm{j})$ montre une insolubilisation significative en présence de macrophages (Tab. I). Les pourcentages dissous à $24 \mathrm{~h}$ sont respectivement de $6,0 \%$ avec macrophages et $17,8 \%$ sans macrophages ; le taux de dissolution lent $s_{\mathrm{s}}$ correspondant à la période de $48 \mathrm{j}$ est de $14,4 \times 10^{-3} \mathrm{j}^{-1}$.

\subsection{Etudes in vivo}

Les études d'inhalation par des rats, dont les résultats sont regroupés dans le tableau II, ont permis, en mesurant la charge pulmonaire dans le temps, de calculer les deux périodes de demi-vie biologique, à savoir $2,3 \mathrm{j}(35 \%)$ et $77 \mathrm{j}$ $(65 \%)$. Le coefficient $F_{\mathrm{b}}$ de transfert a été calculé sur les périodes de $0-7 \mathrm{j}$ et 7-42 j (Tab. III) ainsi que les valeurs dérivées de la CIPR 30 (20) pour les classes $W$ et $Y$. L'excrétion urinaire, exprimée en \% de la quantité initiale déposée, est de $9,5 \%$ à $24 \mathrm{~h}$. Le transfert du sang dans l'urine étant compris entre $54 \%$ (11) et $67 \%$ (22), la quantité dissoute du poumon dans le sang à $24 \mathrm{~h}$ serait donc comprise entre 14 et $19 \%$. L'observation au MEBT des macrophages prélevés après dépôt intratrachéal du composé $\mathrm{UO}_{2}+\mathrm{U}_{\text {métal }}$ 


\section{TABLEAU I}

Résultats de solubilité in vitro du composé $\mathrm{UO}_{2}+\mathbf{U}_{\text {métal }}$ exprimés en pourcentage d'uranium dissous avec le temps en test chimique (milieu de culture seul) ou en test cellulaire (avec macrophages) In vitro solubilities of $\mathrm{UO}_{2}+U_{\text {metal }}$ expressed in $\mathrm{U}$ percentage dissolved through time - chemical test (culture medium only) or cellular test (macrophages)

\begin{tabular}{|ccc|}
\hline Temps & \% deuramium dissous & \% \\
(jours) & $\begin{array}{c}\text { Test cellulaire } \\
\text { avec macrophages }\end{array}$ & $\begin{array}{c}\text { Test chimique } \\
\text { milieu de culture seul }\end{array}$ \\
\hline 1 & $6,0 \pm 2,3(n=85)$ & $17,8 \pm 5,8(n=115)$ \\
2 & $3,4 \pm 1,7(n=59)$ & $9,1 \pm 3,2(n=95)$ \\
3 & $3,4 \pm 1,2(n=32)$ & $5,4 \pm 2,3(n=95)$ \\
\hline
\end{tabular}

$n=$ nombre d'essais.

\section{TABLEAU II}

Distribution dans les organes et excrétion urinaire cumulée après inhalation par des rats du composé $\mathbf{U O}_{2}+\mathbf{U}_{\text {métal }}$

Organ distribution and cumulated urinary excretion following inhalation of $\mathrm{UO}_{2}+\mathrm{U}_{\text {metal }}$ by rats

\begin{tabular}{|c|c|c|c|c|c|}
\hline \multicolumn{6}{|c|}{ 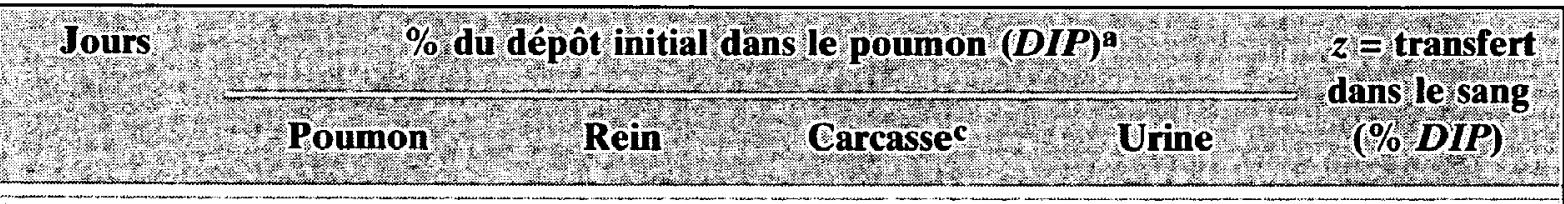 } \\
\hline 3 & $77,5(3,5) b$ & $3,3(1,5)$ & $1,6(0,6)$ & $16,5(4,0)$ & 21,4 \\
\hline 7 & $64,0(4,0)$ & $2,9(0,5)$ & $2,8(0,6)$ & $23,0(4,0)$ & 28,7 \\
\hline 14 & $59,5(9,0)$ & $1,1(0,4)$ & $3,8(0,8)$ & $29,0(7,0)$ & 33,9 \\
\hline 21 & $52,0(8,5)$ & $0,6(0,3)$ & $4,0(0,8)$ & $33,0(9,0)$ & 37,6 \\
\hline 28 & $49,0(3,5)$ & $0,5(0,2)$ & $2,5(0,7)$ & $34,5(4,0)$ & 37,5 \\
\hline 42 & $44,5(4,0)$ & $0,4(0,2)$ & $2,3(0,6)$ & $36,0(5,0)$ & 38,7 \\
\hline
\end{tabular}

a $: D I P=24,4(6,4) \mu \mathrm{g}$.

b : ( ) écart-type pour $n=3$ animaux.

c : estimée à partir de valeurs obtenues pour le fémur.

révèle, en utilisant la raie $\mathrm{L}_{2,3}$ du phosphore à $132 \mathrm{eV}$, la présence d'aiguilles de phosphate d'uranyle (Fig. 3) identifiées par spectrométrie de perte d'énergie des électrons. 


\section{E. ANSOBORLO et al.}

\section{TABLEAU III}

Taux de transfert de l'uranium, du poumon dans le sang après inhalation du composé $\mathrm{UO}_{2}+\mathrm{U}_{\text {métal }}$ par des rats, comparé aux valeurs dérivées de la CIPR 30 pour les composés de classe $W$ et $Y$

$U$ transfer rates within a given time, from lung to blood following inhalation of $\mathrm{UO}_{2}+\mathrm{U}_{\text {metal }}$ by rats, vs ICRP 30 derived values for compounds of class $W$ and $Y$

\begin{tabular}{|lcc|}
\hline Composé & Taux de transfert $F_{\mathbf{b}}^{\mathbf{a}}\left(\mathbf{j}^{-1}\right)$ \\
\hline & $\mathbf{0 - 7} \mathbf{j}$ & $\mathbf{7 - 4 2 \mathbf { j }}$ \\
\hline $\mathrm{UO}_{2}+\mathrm{Y}_{\text {métal }}$ & $41 \times 10^{-3}$ & $7,0 \times 10^{-3}$ \\
\hline $\mathrm{Class} \mathrm{W}$ & $47 \times 10^{-3}$ & $5,1 \times 10^{-3}$ \\
\hline Class $\mathrm{Y}$ & $2,4 \times 10^{-3}$ & $0,16 \times 10^{-3}$ \\
\hline
\end{tabular}

Le taux de transfert $F_{\mathrm{b}}$ est défini par l'équation suivante :

$$
F_{\mathrm{b}}=\frac{0,693}{t_{1 / 2}} \times \frac{z}{y} \mathrm{j}^{-1}
$$

où, sur un interval de temps donné, $t_{1 / 2}$ est le temps de demi-vie dans le poumon, $z$ est le pourcentage de dépôt initial qui passe dans le sang et y le pourcentage de dépôt initial éliminé du poumon.

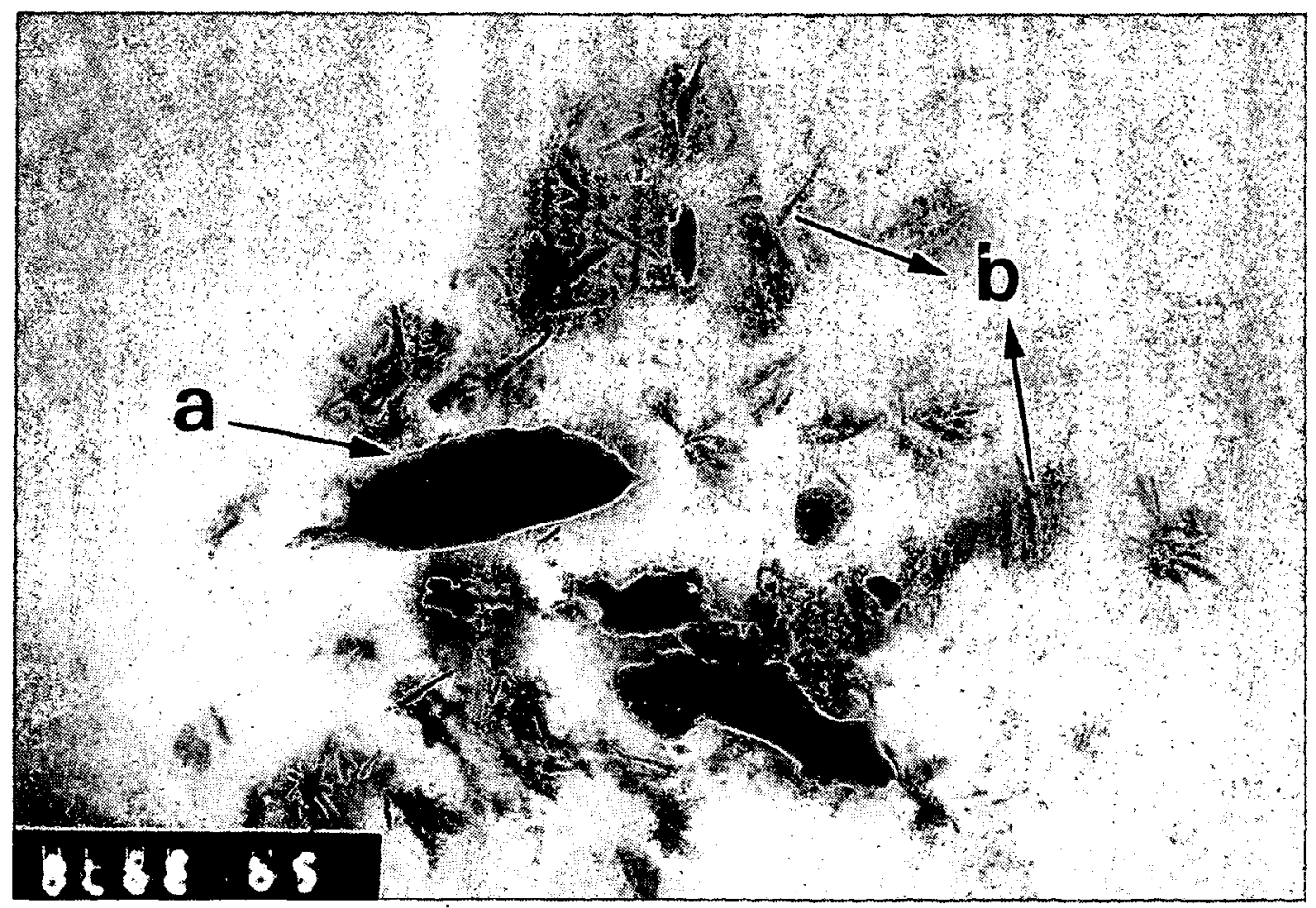

Fig. 3 - Particules d'uranium dans un lysosome $(G=55000)$; a) particule non transformée d'U $\mathrm{O}_{2}+U_{\text {métal }}$; $b$ ) aiguilles de phosphates d'uranyle.

$U$ particles within a lysosome $(G=55000)$; a) $U O_{2}+U_{\text {metal }}$ untransformed particle; b) uranyl phosphate needles. 


\subsection{Paramètres obtenus pour le calcul de dose}

Les paramètres spécifiques obtenus au cours de cette étude sont donnés dans le tableau IV et les valeurs de fractions ou taux de dissolution sont comparées aux valeurs par défaut proposées par le modèle en terme de paramètres d'absorption dans le sang de type $F, M$ et $S$ et tels qu'ils sont utilisés pour le calcul de dose [6].

\section{TABLEAU IV}

Valeurs par défaut et résultats expérimentaux d'absorption sanguine suivant les recommandations de la nouvelle CIPR 66 Calculated and experimental values of blood uptake according to the new ICRP 66

\begin{tabular}{|ccccc|}
\hline \multicolumn{1}{c}{ Type } & In vitro & In vivo & $M^{*}$ & $S^{*}$ \\
\hline $\begin{array}{l}\text { Paramètres CIPR 66 } \\
\cdots\end{array}$ & & & & \\
\hline $\begin{array}{l}f_{\mathrm{r}}: \text { fraction éliminée } \\
\text { par dissolution rapide (\%) }\end{array}$ & 2,6 & 35 & 10 & 0,1 \\
\hdashline $\begin{array}{l}s_{\mathrm{r}}: \text { taux de dissolution } \\
\text { rapide }\left(\mathrm{j}^{-1}\right)\end{array}$ & 0,7 & $41 \times 10^{-3}$ & 100 & 100 \\
\hdashline $\begin{array}{l}s_{\mathrm{r}}: \text { taux de dissolution } \\
\text { lente }\left(\mathrm{j}^{-1}\right)\end{array}$ & $14,4 \times 10^{-3}$ & $7,0 \times 10^{-3}$ & $5,0 \times 10^{-3}$ & $1,0 \times 10^{-4}$ \\
\hline
\end{tabular}

* $M$ et $S$ sont les types d'absorption sanguine par défaut définis dans la CIPR 66 .

\section{Discussion}

L'évaluation des risques radiotoxicologiques sur les installations impliquées dans le développement du nouveau procédé d'enrichissement de l'uranium par laser nous a conduit à étudier la physico-chimie des poussières manipulées aux postes HORUS et ARUS, et a contribué à l'étude de leur comportement biologique in vitro et in vivo. Les résultats ont permis de déterminer un certain nombre de paramètres (concentration, $D A M A$, périodes biologiques) permettant d'une part de connaître la transférabilité et d'autre part de mieux comprendre certains mécanismes régissant le comportement biologique du composé $\mathrm{UO}_{2}+\mathrm{U}_{\text {métal }}$ généré.

Les résultats des mesures de concentration atmosphérique montrent une variation journalière importante due au mode de manipulation et aux problèmes techniques rencontrés à cette phase de développement du procédé pilote. L'augmentation de concentration mesurée à la source lors de l'opération de grattage semble bien corrélée avec des valeurs plus importantes au niveau individuel. La distribution granulométrique conduisant au DAMA 
révèle deux types de classes : une classe de 5,2 à $10 \mu \mathrm{m}$ normalement rencontrée sur ce type de poste, et une classe plus fine correspondant à des amas de particules de diamètre compris entre 50 et 100 nm qui apparaissent plus particulièrement lors de l'initialisation de petits feux d'uranium.

La solubilité chimique révèle une composante rapide de $1 \mathrm{j}$ comme pour tous les composés de l'uranium déjà étudiés $[1-2,4]$ et une composante plus lente de $48 \mathrm{j}$, représentative d'un composé moyennement soluble de type $\mathrm{W}$ suivant la CIPR 30. D'autre part, la diminution de solubilité due à l'action des macrophages confirme certains résultats déjà observés sur $\mathrm{U}_{3} \mathrm{O}_{8}$ par Poncy [15] et sur $\mathrm{UO}_{3}$ par Ansoborlo [4]. Ce phénomène peut être expliqué par la précipitation intracellulaire du composé $\mathrm{UO}_{2}+\mathrm{U}_{\text {métal }}$ sous forme d'aiguilles de phosphate d'uranyle insoluble, et serait dû à l'action enzymatique des lysosomes (principalement la phosphatase acide) aussi bien sur une particule phagocytée que sur l'ion uranyle pénétrant le macrophage comme l'ont montré Galle [9] et Berry [5].

L'expérimentation in vivo confirme les résultats observés in vitro avec une cinétique de décroissance pulmonaire à deux composantes, une rapide de $2,3 \mathrm{j}$ et une plus lente de $77 \mathrm{j}$. Cette période biologique supérieure à celle de $48 \mathrm{j}$ obtenue avec le test in vitro chimique peut s'expliquer en tenant compte, en plus du phénomène de solubilité chimique, du mécanisme de précipitation intracellulaire ainsi que de l'épuration mécanique.

La proximité des résultats de dissolution obtenus à $t=1 \mathrm{j}$ soit in vitro avec $6 \%$ de macrophages et $17,8 \%$ sans macrophages, soit in vivo en considérant la dissolution du poumon vers le sang estimée entre 14 et $19 \%$, montre une bonne corrélation entre les deux tests. Cette corrélation est confirmée en comparant les résultats (Tab. III et IV) exprimés en taux de transfert $F_{\mathrm{b}}$ pour la période de dissolution la plus lente, assimilé au terme $s_{\mathrm{s}}$ du nouveau modèle pulmonaire : en effet, les valeurs obtenues pour $F_{\mathrm{b}}$ sont respectivement de $14,4 \times 10^{-3} \mathrm{j}^{-1}$ in vitro et $7 \times 10^{-3} \mathrm{j}^{-1}$ in vivo et sont comparables à ceux dérivés de la CIPR 30 pour un composé W soit $5,1 \times 10^{-3} \mathrm{j}^{-1}$.

Ces résultats sont assez proches, en terme de période biologique, des données obtenues sur des fumées d'uranium métal avec des périodes de 7 j (40\%) et $70 \mathrm{j}(53 \%)$ observées chez l'homme [17], et en terme de $F_{\mathrm{b}}$, supérieurs aux valeurs obtenues par Stradling [20] pour un $\mathrm{U}_{3} \mathrm{O}_{8}$ formé lors d'une opération de fonte avec $10^{-3} \mathrm{j}^{-1}$, ce qui correspond à une dissolution plus importante pour le composé $\mathrm{UO}_{2}+\mathrm{U}_{\text {métal}}$.

L'ensemble des résultats ainsi obtenus montrent que, contrairement à l'oxyde $\mathrm{UO}_{2}$, classé comme un composé peu transférable $[14,19]$, le mélange $\mathrm{UO}_{2}+\mathrm{U}_{\text {métal }}$ a un comportement moyennement transférable de type $\mathrm{W}$ (CIPR 30) ou M (CIPR 66) [11-12].

Une étude particulière en cours, concernant le cas des feux d'uranium qui conduisent d'une part à l'apparition de particules très fines $(<0,1 \mu \mathrm{m})$ et 
d'autre part à la formation d' $\mathrm{U}_{3} \mathrm{O}_{8}$, permettra, en complément des paramètres spécifiques obtenus pour le composé $\mathrm{UO}_{2}+\mathrm{U}_{\text {métal }}$, et regroupés dans le tableau IV, de faire un calcul de dose efficace et ainsi de déterminer des limites annuelles d'incorporation (LAI) et des limites de concentration admissible (LDCA) qui seront nécessaires pour la surveillance du procédé SILVA dans sa phase d'exploitation.

\section{Conclusion}

Nous avons essayé de montrer, à travers cette étude, l'utilité d'une méthodologie allant du prélèvement d'aérosols au poste de travail jusqu'à la détermination des paramètres de comportement biologique, en tenant compte des propriétés physico-chimiques du composé ainsi que de sa biocinétique. L'application au procédé pilote d'enrichissement de l'uranium par laser nous a permis

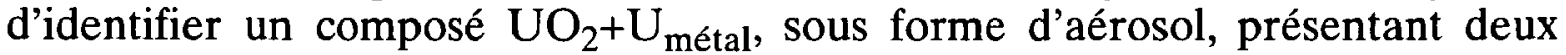
classes de granulométrie dont une plus fine dans le cas de fumées d'uranium. Ces résultats ont permis de déterminer que le composé $\mathrm{UO}_{2}+\mathrm{U}_{\text {métal }}$ est de type W selon la classification de la CIPR 30 et de connaître tous les paramètres spécifiques, dont plus particulièrement les taux de dissolution journalier définis dans la CIPR 66, et qui permettront d'effectuer des calculs de dose efficace. D'autre part, la cohérence des résultats in vitro et in vivo montre l'intérêt de la poursuite simultanée de ce type d'expérimentation.

\section{Remerciements}

Les auteurs tiennent à remercier les équipes d'HORUS et ARUS de la Direction du cycle du combustible du Commissariat à l'énergie atomique (CEA/DCC).

\section{RÉFÉRENCES}

[1] ANSOBORLO E., BERARD P., CHALABREYSSE J. - Study of industrial exposure to class $\mathrm{Y}$ uranium compounds : methods and results. Radiat. Prot. Dosim., 1989, 26, 101-105.

[2] ANSOBORLO E., GIBERT B., CHALABREYSSE J. - Influence des propriétés physico-chimiques du tétrafluorure d'uranium sur les modalités de la surveillance de l'exposition chronique à ce composé. Radioprotection, 1990, 25, 225-234.

[3] ANSOBORlo E., CHAlabReysSe J., HENGE-NAPOli M.H., PUJOl E. Methodology for uranium compounds characterization applied to biomedical monitoring. J. Radioanal. Nucl. Chem., 1992, 161, 79-87.

[4] ANSOBORLO E., CHALABREYSSE J., HENGE-NAPOLI M.H. - In vitro chemical and cellular tests applied to uranium trioxide with different hydration states. Environ. Health Perspect., 1992, 97, 139-143.

[5] BERRY J.P., MEIGNAN M., ESCAIG F., GALLE P. - Inhaled soluble aerosols insolubilised by lysosomes of alveolar cells, application to some toxic compounds : electron microprobe and ion microprobe studies. Toxicology, 1988, 52, 127-139. 
[6] BIRCHALL A., BAILEY M.R., JAMES A.C. - LUDEP : a lung dose evaluation program. Radiat. Prot. Dosim., 1991, 38, 167-174 et Radioprotection 1994, 29 (1) 81 86.

[7] COOKE H., HOLT F.B. - The solubility of some uranium compounds in simulated lung fluid. Health Phys., 1974, 27, 69-77.

[8] EIDSON A.F., DAMON E.G., HAHN F.F., GRIFFITH W.C. - The utility of in vitro solubility testing in assessment of uranium exposure. Radiat. Prot. Dosim., 1989, 26, 69-74.

[9] GALLE P. BERRY J.P., GALLE C. - Role of alveolar macrophages in precipitation of mineral elements inhaled as soluble aerosols. Environ. Health Perspect., $1992,97,145-147$.

[10] HYATT E.C., MOSS W.D., SCHULTE H.F. - Particle size studies on uranium aerosols from machining and metallurgy operations. Ind. Hyg. J., 1959, 99-107.

[11] INTERNATIONAL COMMISSION ON RADIOLOGICAL PROTECTION (ICRP) - Limits for intakes of radionuclides by workers, Pt 1. (ICRP Publication 30). Oxford : Pergamon press. Ann. ICRP, 1979, 2 (3/4).

[12] INTERNATIONAL COMMISSION ON RADIOLOGICAL PROTECTION (ICRP) - Human respiratory tract model for radiological protection. (ICRP Publication 66). Oxford : Pergamon press. Ann. ICRP, 1994, 24 (1/4).

[13] KALKWARF D.R. - Dissolution rates of uranium compounds in simulated lung fluids. Sci. Total Environ., 1983, 28, 405-414.

[14] MÉTIVIER H., PONCY J.L., RATEAU G., STRADLING G.N. - Uranium behaviour in the baboon after the deposition of a ceramic form of uranium dioxide and octoxide in the lungs : implications for human exposure. Radioprotection, 1992, 27, 263-2181.

[15] PONCY J.L., MÉTIVIER H., DHILLY M., VERRY M., MASSE R. - In vitro dissolution of uranium oxide by baboon alveolar macrophages. Environ. Health Perspect., 1992, 97, 127-130.

[16] SCHIEFERDECKER H., DILGER H., DOERFEL H. - Inhalation of uranium aerosols from $\mathrm{UO}_{2}$ fuel element fabrication. Health Phys., 1985, 48, 29-48.

[17] SCOTT L.M., WEST C.M. - An evaluation of $\mathrm{U}_{3} \mathrm{O}_{8}$ exposure with an estimate of systemic body burden. Health Phys., 1967, 13, 21-26.

[18] STRADLING G.N., STATHER J.W., GRAY S.A. - The metabolic behaviour of $\mathrm{U}_{3} \mathrm{O}_{8}$ bearing residues after their deposition in the rat lung : the implications for occupational exposure. Exp. Pathol., 1989, 37, 76-82.

[19] STRADLING: G.N., STATHER J.W., GRAY S.A. - The metabolism of ceramic and non ceramic forms of $\mathrm{UO}_{2}$ after deposition in the rat lung. Human Toxicol., 1988, 7, 133-139.

[20] STRADLING G.N., STATHER J.W., COOKE N. - Metabolism of uranium in the rat after inhalation of two industrial forms of ore concentrate : the implications for occupational exposure. Human Toxicol., 1987, 6, 385-393.

[21] TASAT D.R., DE REY B.M. - Cytotoxic effect of $\mathrm{UO}_{2}$ on rat alveolar macrophages. Environ. Res., 1987, 44, 71-81.

[22] WRENN M.E., BERTELLI L., DURBIN P.W., ECKERMAN K.F. - A comprehensive metabolic model for uranium metabolism and dosimetry based on human and animal data. In : Intakes of radionuclides, detection, assessment and limitation of occupational exposure, Bath, 13-17 september 1993 (J.W. Stather, A. Karaoglou, Eds). Radiat. Prot. Dosim., 1994, 53 (1-4) 255-261. 